\title{
Bose Condensation and the BTZ Black Hole
}

\author{
Cenalo Vaz ${ }^{a, b, 1}$, L.C.R. Wijewardhana ${ }^{b, 2}$ \\ ${ }^{a} R W C$ and ${ }^{b}$ Department of Physics, \\ University of Cincinnati, \\ Cincinnati, Ohio 45221-0011, USA
}

\begin{abstract}
Although all popular approaches to quantum gravity are able to recover the BekensteinHawking entropy-area law in the thermodynamic limit, there are significant differences in their descriptions of the microstates and in the application of statistics. Therefore they can have significantly different phenomenological implications. For example, requiring indistinguishability of the elementary degrees of freedom should lead to changes in the black hole's radiative porperties away from the thermodynamic limit and at low temperatures. We demonstrate this for the Bañados-Teitelboim-Zanelli (BTZ) black hole. The energy eigenstates and statistical entropy in the thermodynamic limit of the BTZ black hole were obtained earlier by us via symmetry reduced canonical quantum gravity. In that model the BTZ black hole behaves as a system of Bosonic mass shells moving in a one dimensional harmonic trap. Bose condensation does not occur in the thermodynamic limit but this system possesses a finite critical temperature, $T_{c}$, and exhibits a large condensate fraction below $T_{c}$ when the number of shells is finite.
\end{abstract}

PACS 04.60.Ds, 04.70.Dy, 03.75.Lm

\footnotetext{
${ }^{1}$ e-mail address: Cenalo.Vaz@UC.Edu

2 e-mail address: Rohana.Wijewardhana@UC.Edu
} 


\section{INTRODUCTION}

A solution to the problem of explaining black hole thermodynamics [1] 3] from a canonical or microcanonical ensemble can be expected to provide important insights into quantum gravity, which is considered to be a central problem of theoretical physics. However, there is no general consensus on the true nature of the elementary degrees of freedom because many apparently different approaches to quantum gravity have all successfully recovered the Bekenstein-Hawking entropy.

The differences between the approaches appear fundamental and range from their intrinsic description of the quantum gravity states to the statistics used in counting them. For example, in string theory the microstates are dual to weak field D-brane states [4], in the AdS/CFT approach they are taken to be the states of a particular horizon conformal field theory (CFT) [0 11] and in loop quantum gravity (LQG) they are represented by punctures of a spin network on the event horizon [12 17. It seems unlikely that these descriptions are related in some as yet unknown way because of a more subtle distinction between them, which is their use of statistics. In string theory and AdS/CFT the elementary degrees of freedom are indistinguishable whereas they must be treated as distinguishable in LQG in order to recover the Bekenstein-Hawking law. The statistical properties of the microstates can have far ranging phenomenological implications [18], particularly at low temperatures and outside the thermodynamic limit.

In this paper we ask what behavior one may expect outside the thermodynamic limit. Such a question can only be answered in a model dependent way, therefore we pose it within the context of a model of the quantum black hole that arises from canonical quantum gravity applied to spherical gravitational collapse [19]. In this model a black hole microstate is viewed as a particular distribution of matter shells, which have crossed the horizon, among the available black hole energy levels. This is a natural way to realize Bekenstein's original ideas in [1], from which we quote: "It is then natural to introduce the concept of black hole entropy as the measure of the inaccessibility of information (to an exterior observer) as to which particular internal configuration of the black hole is actually realized in a given case."

The simplest example is provided by the static Bañados-Teitelboim-Zanelli (BTZ) black hole [20]. A symmetry reduced canonical quantization of this black hole yields Hawking radiation 21] and an equispaced black hole mass spectrum. Furthermore, counting the black hole's microstates in the canonical ensemble reproduces the Bekenstein-Hawking entropy in the thermodynamic limit, provided that the counting proceeds using Bose statistics [22]. The thermodynamic limit is the limit in which both the system size and energy are simultaneously taken to be very large. For the BTZ black hole this means that the temperature is high. Our interest here is to examine the same ensemble at low temperatures and for a finite number of matter shells. We will show that Bose-Einstein condensation occurs and the BTZ black hole turns "cold" once condensation sets in. "Cold" black holes are of course stable against Hawking evaporation.

One may criticize our choice of the $2+1$ dimensional black hole, even though it has the virtue of being simple, because it is topological and therefore unique in many respects. For instance when canonical quantum gravity is applied to the Schwarzschild or higher dimensional black holes in the absence of a cosmological constant the microstates are counted 
in an "area ensemble" using Boltzmann statistics. However the situation is different for "large" Anti-de Sitter (AdS) black holes, which are black holes whose horizon radius is much larger than the AdS length. In a particular limit, their mass spectra are identical to the spectrum of the BTZ black hole modulo dimension dependent constant factors and Bose statistics must be applied to recover the Bekenstein-Hawking area law [23]. Moreover, many higher dimensional black holes from string theory have the form BTZ $\times \mathcal{M}$, where $\mathcal{M}$ is a simple manifold. Their thermodynamic properties can be recovered directly from the thermodynamic properties of the BTZ black hole [24, 25]. Bose condensation is a possibility whenever indistinguishability is an essential feature of the counting of microstates. It may thus occur for realistic black holes in higher dimensions, in which case one can expect stable black hole remnants to contribute significantly to the Dark Matter content of the universe.

This paper is organized as follows. In section II we review the canonical quantization of the BTZ black hole and its statistical thermodynamics in the canonical ensemble, as given in [21]. We then go outside the thermodynamic limit in section III, treating the black hole as a system of finite size. With the black hole spectrum, which we derive in section II, we show that Bose condensation can occur. We go on to determine the critical temperature and the condensate fraction as well as the entropy and argue that the area law strictly holds only in the thermodynamic limit but breaks down outside it and below the critical temperature. We conclude in section IV with some comments.

\section{STATISTICAL THERMODYNAMICS OF THE BTZ BLACK HOLE}

The static BTZ black hole may be viewed as the end state of the collapse of inhomogeneous dust [26, 27], which is described by the LeMaître-Tolman-Bondi family of solutions of Einstein's equations with a negative cosmological constant. The solutions are characterized by two arbitrary functions, viz., the "mass function", $F(\rho)=4 G M(\rho)$, representing the initial mass distribution of the dust ball and the energy function, $E(\rho)$ representing its initial energy distribution. The classical solutions are given by the circularly symmetric line element

$$
d s^{2}=d \tau^{2}-\frac{\left(\partial_{\rho} R\right)^{2}}{2(E-F)} d \rho^{2}-R^{2} d \varphi^{2}
$$

where $\tau$ is the dust proper time and $\rho$ labels dust shells or curvature radius $R(\tau, \rho)$. Einstein's equations can be integrated to give the energy density of the dust

$$
\varepsilon(\tau, \rho)=\frac{\partial_{\rho} F}{R\left(\partial_{\rho} R\right)}
$$

and a dynamical equation for the evolution of the area radius,

$$
\left(\partial_{\tau} R\right)^{2}=2 E-\Lambda R^{2}
$$

in terms of two arbitrary integration functions of the shell index coordinate, $\rho$. The last determines the area radius of shells to be

$$
R(\tau, \rho)=\sqrt{\frac{2 E}{\Lambda}} \sin \left(-\sqrt{\Lambda} \tau+\sin ^{-1} \sqrt{\frac{\Lambda}{2 E}} \rho\right),
$$


where we have used a freedom in the scaling of the shell index $\rho$ by setting $R(0, \rho)=\rho$ and the fact that collapse solutions satisfy $\partial_{\tau} R<0$. The integration function $F(\rho)$ represents the initial mass distribution of the collapsing dust ball and the function $E(\rho)$ represents the initial energy distribution; one finds

$$
\begin{aligned}
& F(\rho)=\int_{0}^{\rho} \varepsilon(0, \rho) \rho d \rho \\
& E(r)=\frac{1}{2}\left[\left(\partial_{\tau} R\right)_{\tau=0}^{2}+\Lambda R^{2}\right] .
\end{aligned}
$$

directly from (2) and (3) respectively.

The general circularly symmetric Arnowitt-Deser-Misner (ADM) line element,

$$
d s^{2}=N^{2} d t^{2}-L^{2}\left(d r-N^{r} d t\right)^{2}-R^{2} d \varphi^{2}
$$

can be embedded into the metric in (何). After a series of transformations described in detail in 21] this leads to a canonical description of the classical black hole in terms of the dust proper time, $\tau(r)$, the area radius, $R(r)$, the mass density defined via

$$
F(r)=\frac{M_{0}}{2}+\int_{0}^{r} d r^{\prime} \Gamma(r)
$$

where $M_{0}$ is an arbitrary constant contributed by the boundary at the origin, and their conjugate momenta, $P_{\tau}(r), P_{R}(r)$ and $P_{\Gamma}(r)$ respectively. The effective constraints of the gravity-dust system are then obtained in the form

$$
\begin{aligned}
\mathcal{H}_{r} & =\tau^{\prime} P_{\tau}+R^{\prime} P_{R}-\Gamma P_{\Gamma}^{\prime} \approx 0 \\
\mathcal{H} & =P_{\tau}^{2}+\mathcal{F} P_{R}^{2}-\frac{\Gamma^{2}}{\mathcal{F}} \approx 0,
\end{aligned}
$$

where $\mathcal{F}=\Lambda R^{2}-F$ and the prime refers to derivatives with respect to the ADM label coordninate $r$.

Applying Dirac's formal quantization to the above system of constraints, we replace the momenta by functional derivatives with respect to their corresponding configuration variables. To encapsulate the factor ordering ambiguities at the formal level of the WheelerDeWitt equation we introduce factors of $\delta(0)$ into the resulting functional Schroedinger equation, writing the quantum Hamiltonian constraint as

$$
\widehat{\mathcal{H}} \Psi[\tau, R, \Gamma]=\left[\frac{\delta^{2}}{\delta \tau^{2}}+\mathcal{F} \frac{\delta^{2}}{\delta R^{2}}+A \delta(0) \frac{\delta}{\delta R}+B \delta(0)^{2}+\frac{\Gamma^{2}}{\mathcal{F}}\right] \Psi[\tau, R, \Gamma]=0,
$$

where $A(R, F)$ and $B(R, F)$ are smooth functions. The continuum limit of the wavefunctional is taken to be of the form

$$
\Psi[\tau, R, \Gamma]=\exp \left[i \int d r \Gamma(r) \mathcal{W}(\tau(r), R(r), F(r))\right],
$$

which formally obeys the momentum constraint provided that $\mathcal{W}(\tau, R, F)$ has no explicit dependence on the label coordinate $r$. We then put (9) and (10) on a lattice and take the 
continuum limit [28]. This corresponds to the choice of regularization. Consistency of the lattice regularization fixes the factor ordering via a set of three equations [29] for each lattice site, $j$,

$$
\begin{aligned}
& {\left[\left(\frac{\partial \mathcal{W}_{j}}{\partial \tau_{j}}\right)^{2}+\mathcal{F}_{j}\left(\frac{\partial \mathcal{W}_{j}}{\partial R_{j}}\right)^{2}-\frac{1}{\mathcal{F}_{j}}\right]=0} \\
& {\left[\frac{\partial^{2} \mathcal{W}_{j}}{\partial \tau_{j}^{2}}+\mathcal{F}_{j} \frac{\partial^{2} \mathcal{W}_{j}}{\partial R_{j}^{2}}+A_{j} \frac{\partial \mathcal{W}_{j}}{\partial R_{j}}\right]=0} \\
& B_{j}=0
\end{aligned}
$$

Thus $B(R, F)$ is constrained to be identically vanishing and $A(R, F)$ and the lattice wavefunctions are obtained by solving the first two equations. Furthermore, hermiticity of the Hamiltonian constraint requires that the Hilbert space measure $\mathfrak{m}(R, F)$ is determined from $A(R, F)$ according to

$$
A_{j}=\left|\mathcal{F}_{j}\right| \partial_{R_{j}} \ln \left(\mathfrak{m}_{\mathfrak{j}}\left|\mathcal{F}_{j}\right|\right)
$$

Thus, the system is completely solved once regularized on the lattice.

Unfortunately, the situation becomes more complicated when the mass density function is distributional, as it is in the description of the final state black hole, for then the system naturally collapses into a countable product of wave functions, the functional differential equations become ordinary partial differential equations and no regularization is required. It follows that no further conditions are available, leaving the factor ordering ambiguity in the form of the unknown functions $A$ and $B$ unresolved.

This is exemplified by the BTZ black hole whose metric is of the form

$$
d s^{2}=-\left(\Lambda R^{2}-8 G M\right) d T^{2}+\frac{d R^{2}}{\left(\Lambda R^{2}-8 G M\right)}+R^{2} d \varphi^{2},
$$

where $M$ is the black hole mass parameter. This is a special solution of (1) in which the mass function is taken to be constant, $F=4 G M$, for $\rho>0$ and the energy function is given by $2 E=1+8 G M$, again for $\rho>0$. The metric in (11) can be brought to the static form in (13) by the transformations $R=R(\tau, \rho)$ as given in (44) and

$$
T=\tau+\int d R \frac{\sqrt{1+8 G M-\Lambda R^{2}}}{\Lambda R^{2}-8 G M}
$$

for the Killing time, $T$. Within the framework of the canonical theory we take

$$
F(r)=4 G M_{0}+4 G \varepsilon \Theta(r)=4 G M
$$

where $\varepsilon$ represents the mass of a shell at $r=0$ and where $\Theta$ is the Heaviside function. Likewise, the energy function should be given by

$$
E(r)=\frac{1}{2}\left[1+8 G M_{0}+8 G \varepsilon \Theta(r)\right]
$$

We see that the mass function in (15) yields a mass density that is the $\delta$-distribution

$$
\Gamma(r)=4 G \varepsilon \delta(r)
$$


and the wave-functional in (10) turns into the wave-function,

$$
\Psi=e^{\frac{i}{4 G} \int_{0}^{\infty} d r \Gamma(r) \mathcal{W}(\tau(r), R(r), F(r))}=e^{i \varepsilon \mathcal{W}(\tau, R, F),}
$$

where $\tau=\tau(0), R=R(0)$ and $F=F(0)$. The Wheeler-DeWitt equation becomes

$$
\left[\frac{\partial^{2}}{\partial \tau^{2}}+\mathcal{F} \frac{\partial^{2}}{\partial R^{2}}+A \frac{\partial}{\partial R}+B\right] e^{i \varepsilon \mathcal{W}(\tau, R, F)}=0
$$

and we note that $A(R, F)$ and $B(R, F)$ remain undetermined, as does the measure $\mathfrak{m}(R, F)$, with $B(R, F)$ playing the role of an external potential.

Fortunately there is a way out of this difficulty, which is provided by a detailed examination of the Hawking radiation from the black hole within the context of this midisuperspace model. Because the Wheeler DeWitt equation is second order in the dust proper time we can define both positive and negative frequency states with respect to $\tau$. Therefore, taking the collapsing dust as a small perturbation around a pre-existing, massive black hole, so that the role of the quantum matter in Hawking's original background field derivation of black hole radiance [3] is played by the dust, the system (11) can be shown to yield Hawking radiation at the Hawking temperature [21]. However, an important point is that obtaining the correct Planckian distribution in the near horizon limit requires a particular choice of measure, one appropriate to the massive black hole because the dust itself is considered a perturbation on the black hole background. The measure turns out to be the one obtained from the deWitt supermetric, which, from (8), is

$$
\gamma_{a b}=\left(\begin{array}{ll}
1 & 0 \\
0 & \frac{1}{\mathcal{F}}
\end{array}\right) \text {. }
$$

Thus

$$
\mathfrak{m}=\frac{1}{\sqrt{|\mathcal{F}|}}
$$

and putting this together with the hermiticity requirement in (12) determines $A(R, F)$. Now since the black hole ends up being a single shell in this simple quantum mechanical model, it is reasonable to take the external potential, $B$, to be vanishing. Thus (19) reduces to the free Klein-Gordon equation which is hyperbolic in the interior and elliptic elsewhere. A stationary state solution this equation was shown in [22] to yield the mass levels

$$
\varepsilon_{j}=\frac{\hbar}{l}\left(j+\frac{1}{2}\right)
$$

where $l^{2}=-\Lambda^{-1}$ is the AdS length.

The macroscopic black hole is defined as the end state of the collapse of many, say $\mathcal{N}=\sum_{j} \mathcal{N}_{j}$, matter shells, $\mathcal{N}_{j}$ of which occupy level $j$ of (22). Thus, when a boundary contribution from the origin is included [26, 27] $]^{3}$, the total black hole mass is given as

$$
M=M_{0}+\sum_{j} \frac{\hbar}{l}\left(j+\frac{1}{2}\right) \mathcal{N}_{j} .
$$

\footnotetext{
${ }^{3}$ In more than three dimensions this contribution from the origin is usually set to zero, otherwise it would represent a singular initial configuration. In three dimensions a non-vanishing $M_{0}$ is necessary to allow for a velocity profile that vanishes at the origin. This does not lead to singular initial data and the presence of $M_{0}$ does not lead to a singular initial configuration.
} 
From this point of view, the elementary degrees of freedom are bosonic mass shells and a black hole microstate is a particular distribution of $\mathcal{N}$ shells between the levels in (22).

The statistical thermodynamics, specifically the $\mathcal{N} \rightarrow \infty$ limit, of this model can be treated in the canonical ensemble and is captured by the partition function

$$
Z(\beta)=\sum_{\left\{\mathcal{N}_{1}, \ldots, \mathcal{N}_{j}, \ldots\right\}} g\left(\mathcal{N}_{1}, \ldots, \mathcal{N}_{j}, \ldots\right) \exp \left[-\beta\left(M_{0}+\sum_{j} \varepsilon_{j} \mathcal{N}_{j}\right)\right]
$$

where $\mathcal{N}_{j}$ represents the number of shells excited to level $j$, with mass $\varepsilon_{j}$, and $g\left(\mathcal{N}_{1}, \ldots, \mathcal{N}_{j}, \ldots\right)$ is the degeneracy of states, which we take to be unity so as to implement Bose statistics. The canonical entropy is then obtained from

$$
S_{\mathrm{can}}=[\beta M+\ln Z(\beta)]_{M=-\partial \ln Z / \partial \beta},
$$

where $M$ is the average energy in the canonical ensemble, which we associate with the black hole mass. When the system size is taken to be infinite, the partition function

$$
Z(\beta \hbar / 2 l)=e^{-\beta M_{0}} \prod_{j=0}^{\infty}\left[1-e^{-\frac{\beta \hbar}{2 l}(2 j+1)}\right]^{-1}
$$

is easily evaluated. It may be rewritten, by exploiting the well known duality in [30], as

$$
Z(\beta \hbar / 2 l)=\frac{1}{\sqrt{2}} e^{-\left(\frac{\pi^{2} l}{\beta \hbar}+\frac{\beta \hbar}{8 l}\right)\left(\frac{8 l M_{0}}{\hbar}-\frac{1}{6}\right)}\left[Z\left(4 \pi^{2} l / \beta \hbar\right)\right]^{-1} .
$$

This links the high temperature behavior of our system to its low temperature dynamics. If one assigns a value $\Delta_{0}$ to the ground state energy of the system, taking

$$
Z\left(4 \pi^{2} l / \beta \hbar\right) \approx e^{-\frac{8 \pi^{2} l^{2} \Delta_{0}}{\beta \hbar^{2}}}
$$

then it is then a straightforward exercise to show that

$$
S_{\mathrm{can}}=4 \pi \sqrt{c_{\mathrm{eff}} \frac{l M}{6 \hbar}}
$$

where

$$
c_{\mathrm{eff}}=\frac{1}{2}\left[1-\frac{48 l}{\hbar}\left(M_{0}-\Delta_{0}\right)\right]
$$

depends on two undetermined parameters, the vacuum energy $\Delta_{0}$ and the boundary contribution $M_{0}$. The vacuum energy depends on what one takes to be the ground state of the BTZ solution, for example the choice $\Delta_{0}=-1 / 8 G$ corresponds to the choice of pure $\operatorname{AdS}_{3}$,

$$
d s^{2}=-\left(\frac{R^{2}}{l^{2}}+1\right) d T^{2}+\left(\frac{R^{2}}{l^{2}}+1\right)^{-1} d R^{2}+R^{2} d \varphi^{2}
$$

for the ground state [31]. On the other hand, the presence of the arbitrary contribution, $M_{0}$, from the boundary at the origin must be fixed by some other means, for example by 
comparing (29) to the Bekenstein-Hawking entropy of the BTZ black hole. The BekensteinHawking entropy is just

$$
S_{\mathrm{B}-\mathrm{H}}=\frac{A_{h}}{4 G}=\frac{\pi R_{h}}{2 G \hbar}=\frac{\pi l}{\hbar} \sqrt{\frac{2 M}{G}},
$$

where $A_{h}$ is the horizon area and $R_{h}$ its radius. This in turn implies that

$$
c_{\text {eff }}=\frac{3 l}{2 G \hbar}
$$

and therefore fixes $M_{0}-\Delta_{0}$.

\section{THE GRAND CANONICAL ENSEMBLE}

We now consider a black hole made of a finite number $\mathcal{N}=\sum_{j} \mathcal{N}_{j}$ shells. It is standard practice to then begin with the grand partition function,

$$
\Xi(\varepsilon, \beta)=e^{-\beta M_{0}} \prod_{j}\left(1-e^{-\beta\left(\varepsilon_{\alpha}-\mu\right)}\right)^{-1}
$$

where we introduced a chemical potential $\mu$, which is determined from the constraint

$$
\sum_{j}\left\langle\mathcal{N}_{j}\right\rangle=\mathcal{N}
$$

and where $\varepsilon_{j}$ is the energy of the level $j$. Introducing $\lambda=e^{\beta\left(\mu-\varepsilon_{0}\right)}<1\left(\lambda^{-1}\right.$ is the fugacity) and $\Delta \varepsilon_{j}=\varepsilon_{j}-\varepsilon_{0}$ we then have

$$
\Xi(\mu, \beta)=e^{-\beta M_{0}} \prod_{j} \frac{\lambda}{\lambda-e^{-\beta \Delta \varepsilon_{j}}} .
$$

The average level occupation number is computed directly from $\Xi(\beta, \mu)$,

$$
\left\langle N_{j}\right\rangle=-\frac{1}{\beta} \frac{\partial \ln \Xi}{\partial \varepsilon_{j}}=\frac{1}{e^{\beta \Delta \varepsilon_{j}}-\lambda}
$$

so our condition for determining the chemical potential is

$$
\mathcal{N}=\sum_{j=0}^{\infty} \frac{1}{e^{\beta \Delta \varepsilon_{j}}-\lambda}=\frac{\lambda}{1-\lambda}+\sum_{j=1}^{\infty} \frac{\lambda}{e^{\beta \Delta \varepsilon_{j}}-\lambda} .
$$

The first term on the right represents the ground state occupancy (henceforth, average values will be understood and the angular brackets will be omitted),

$$
N_{0}=\frac{\lambda}{1-\lambda}
$$

and the second represents the number of excited shells,

$$
N_{\mathrm{ex}}=\sum_{j=1}^{\infty} \frac{\lambda}{e^{\beta \Delta \varepsilon_{j}}-\lambda}
$$


The condensate fraction is defined as the ratio of the ground state occupancy to the total number of shells, $f_{C}=N_{0} / \mathcal{N}$.

We will first determine $f_{C}$ as a function of the temperature. For the spectrum of the BTZ black hole in (22), $\Delta \varepsilon_{j}=\hbar j / l$ and therefore, replacing the last sum in (38) by its power series expansion, we can express the total number of shells as

$$
\mathcal{N}=\frac{\lambda}{1-\lambda}+\sum_{j=1}^{\infty} \sum_{r=1}^{\infty} \lambda^{r} e^{-\beta \hbar r j / l}
$$

The most direct way to evaluate the double sum in (41) is to employ the Mellin-Barnes representation of the exponential function,

$$
e^{-\alpha}=\frac{1}{2 \pi i} \int_{\tau-i \infty}^{\tau+i \infty} d t \Gamma(t) \alpha^{-t}
$$

where $\tau \in \mathbb{R}$ and $\operatorname{Re}(\alpha)>0$, to re express it as an integral over the complex plane. This gives

$$
\mathcal{N}=\frac{\lambda}{1-\lambda}+\frac{1}{2 \pi i} \int_{\tau-i \infty}^{\tau+i \infty} d t \frac{\Gamma(t) \zeta(t) \mathrm{Li}_{t}(\lambda)}{(\beta \hbar / l)^{t}}
$$

where $\zeta(t)$ is the zeta function and $\operatorname{Li}_{t}(\lambda)$ is the polylogarithm. For $\lambda<1$ the integral is governed by the simple pole of the $\zeta$-function at $t=1$, therefore

$$
\mathcal{N}=\frac{\lambda}{1-\lambda}+\frac{\operatorname{Li}_{1}(\lambda)}{\beta \hbar / l} \equiv \frac{\lambda}{1-\lambda}-\frac{1}{\beta \hbar / l} \ln (1-\lambda)
$$

Equation (44) can be solved for $\lambda$ in terms of the Lambert function, $\mathcal{W}(x)$,

$$
\lambda(\beta, \mathcal{N})=1-\frac{\beta \hbar / l}{\mathcal{W}\left(\beta \hbar / l e^{(\mathcal{N}+1) \beta \hbar / l}\right)}
$$

and determines the ground state occupancy as well as the condensate fraction,

$$
f_{C}(\beta, \mathcal{N})=\frac{N_{0}}{\mathcal{N}}=\frac{\mathcal{W}\left(\beta \hbar / l e^{(\mathcal{N}+1) \beta \hbar / l}\right)}{\mathcal{N} \beta \hbar / l}-\frac{1}{\mathcal{N}},
$$

in terms of the temperature. It is more illuminating, however, to express the condensate fraction as a function of the dimensionless ratio, $x=T / T_{c}$, where $T_{c}$ is the critical temperature at which all the shells are excited.

To calculate the critical temperature, $T_{c}$, it is necessary to set $\lambda \approx 1$ and $N_{\text {ex }}=\mathcal{N}$, the total number of shells. Once again employing the Mellin-Barnes transformation, this time replacing the polylogarithm function $\left.\operatorname{Li}_{t}(\lambda)\right|_{\lambda \approx 1}$ by the $\zeta$-function, we find [32]

$$
N_{\mathrm{ex}}=\mathcal{N} \approx \frac{1}{2 \pi i} \int_{\tau-i \infty}^{\tau+i \infty} d t \frac{\Gamma(t) \zeta^{2}(t)}{\left(\beta_{c} \hbar / l\right)^{t}}
$$

but the integral now admits a double pole at $t=1$ via the $\zeta$-function and therefore

$$
\mathcal{N} \approx\left(\frac{\Gamma(t)}{(\beta \hbar / l)^{t}}\right)^{\prime}=-\frac{l}{\beta_{c} \hbar} \ln \left(e^{\gamma} \beta_{c} \hbar / l\right)
$$




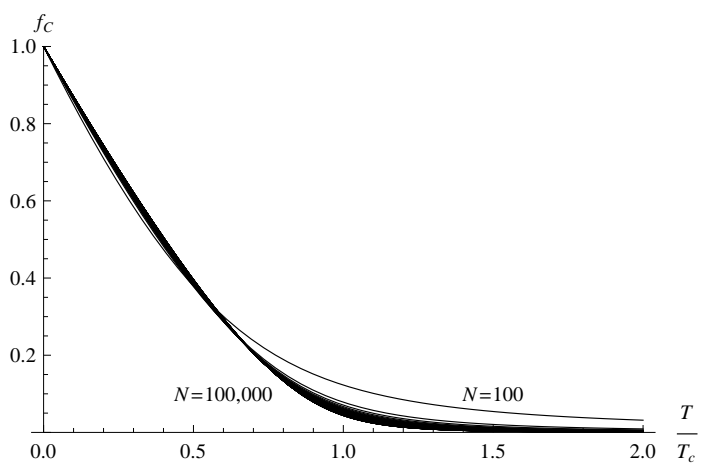

FIG. 1: $f_{C}$, as a function of $T / T_{c}$.

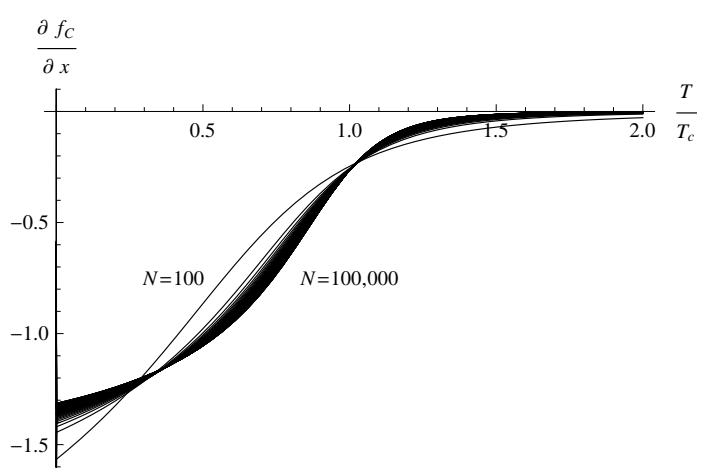

FIG. 2: $\frac{\partial f_{C}}{\partial x}$, as a function of $T / T_{c}$.

where $\gamma$ is the Euler-Mascheroni constant. Solving for the inverse critical temperature, we determine

$$
\beta_{c} \hbar / l=\frac{\mathcal{W}\left(e^{-\gamma} \mathcal{N}\right)}{\mathcal{N}}
$$

which is the exact version of the result in [33]. The Lambert function increases linearly for small values of its argument and slower than the log function for larger values. Thus, for a few shells the critical temperature is more or less constant whereas it increases roughly as $\mathcal{N} / \ln \mathcal{N}$ when $\mathcal{N}$ grows large. This is a well known property of one dimensional harmonic traps. When the black hole is composed of many shells, most of them will in fact lie in the ground state. However, for a small number of shells the ground state occupancy can still be a large percentage of the total number of shells in a significant interval of temperatures.

Using (49) to re-express the condensate fraction in terms of the variable $x=T / T_{c}$, we easily arrive at

$$
f_{C}(x, \mathcal{N})=\frac{x}{\mathcal{W}_{N}} \mathcal{W}\left(\frac{\mathcal{W}_{N}}{\mathcal{N} x} e^{\frac{(\mathcal{N}+1) \mathcal{W}_{N}}{\mathcal{N} x}}\right)-\frac{1}{\mathcal{N}}
$$

where we have defined $\mathcal{W}_{N}=\mathcal{W}\left(e^{-\gamma} \mathcal{N}\right)$. At high temperatures,

$$
f_{C} \stackrel{x \rightarrow \infty}{\approx} \frac{1}{\mathcal{N}}+\frac{\mathcal{W}_{N}}{\mathcal{N} x}+\mathcal{O}\left(x^{-2}\right)
$$

whereas, at low temperatures,

$$
f_{C} \stackrel{x \rightarrow 0}{\approx} 1-\frac{\ln \mathcal{N}}{\mathcal{W}_{N}} x+\mathcal{O}\left(x^{2}\right)
$$

Figure 1 shows the behavior of $f_{C}$ as a function of $x$ for $\mathcal{N}$ ranging from one hundred to one hundred thousand shells. It is small, increasing slowly to the left when $x \gg 1$ but significantly faster when $x \ll 1$ and approaching unity in the limit as $x \rightarrow 0$. Again, the slope of the condensate fraction, $\partial f_{C} / \partial x$, approaches $\ln \mathcal{N} / \mathcal{W}_{N}$ for small $x$ and it is vanishingly small for large $x$. Figure 2 shows this slope for the same range of $\mathcal{N}$. Both the analytical approximations above as well as the figures indicate that increasing the number of shells saturates the slope of the condensate fraction. This behavior suggests that the critical temperature may be thought of as the condensation temperature.

The average energy of the system, which is associated with the black hole mass, can be expressed in terms of the occupancy of the levels in the usual way and the sum rewritten in 


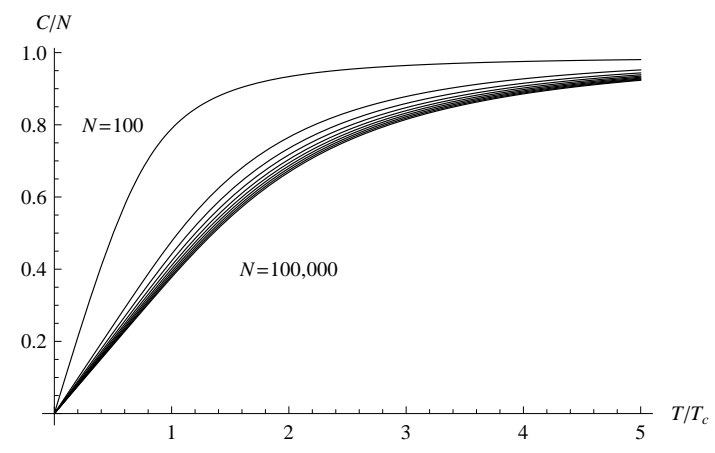

FIG. 3: $C / \mathcal{N}$ as a function of $T / T_{c}$.

the integral representation we have used so far; one gets

$$
M(\beta, \mathcal{N})=M_{0}+\mathcal{N} \varepsilon_{0}+\frac{\hbar / l}{2 \pi i} \int_{\tau-i \infty}^{\tau+i \infty} d t \frac{\Gamma(t) \operatorname{Li}_{t}(\lambda) \zeta(t-1)}{(\beta \hbar / l)^{t}} .
$$

For $\lambda<1$ there is only a simple pole coming from the $\zeta$-function at $t=2$, so the black hole mass can be given in terms of the temperature by

$$
M(\beta, \mathcal{N})=M_{0}+\mathcal{N} \varepsilon_{0}+\frac{\operatorname{Li}_{2}(\lambda)}{\beta^{2} \hbar / l}
$$

and the heat capacity is

$$
C(\beta, \mathcal{N})=\frac{2 \mathrm{Li}_{2}(\lambda)}{\beta \hbar / l}-\frac{\mathrm{Li}_{2}^{\prime}(\lambda)}{\hbar / l}\left(\frac{\partial \lambda}{\partial \beta}\right)_{\mathcal{N}}
$$

where the prime on the polylogarithm represents a derivative with respect to $\lambda$. Its behavior is best understood in terms of the dimensionless parameter $x=T / T_{c}$,

$$
C(x, \mathcal{N})=\frac{2 \mathcal{N} x \mathrm{Li}_{2}(\lambda)}{\mathcal{W}_{N}}-\frac{\mathcal{N} x^{2}}{\lambda \mathcal{W}_{N}} \ln (1-\lambda)\left(\frac{\partial \lambda}{\partial x}\right)_{\mathcal{N}} .
$$

The specific heat, $C / \mathcal{N}$, is displayed as a function of $x=T / T_{c}$ in figure 3 for $\mathcal{N}$ ranging from one hundred to one hundred thousand shells. There is no true phase transition in this system. At low temperatures, the fugacity in (45) behaves roughly as $1-\mathcal{N}^{-1}$ and the second term in the expression for the heat capacity in (56) becomes vanishingly small. Thus in the limit as $\beta \rightarrow \infty$, using $\operatorname{Li}_{2}(1)=\pi^{2} / 6$ we find

$$
\frac{C}{\mathcal{N}} \approx \frac{\pi^{2} k T}{3 \mathcal{N} \hbar / l}
$$

(see [34, 35]). On the other hand, at high temperatures the fugacity behaves as

$$
\lambda=1-e^{-\mathcal{N} \beta \hbar / l}
$$

and the specific heat approaches unity in the limit as $\beta \rightarrow 0$.

We now turn to the entropy, which may be computed from

$$
S=\ln \Xi+\beta U-\mu \beta \mathcal{N}
$$




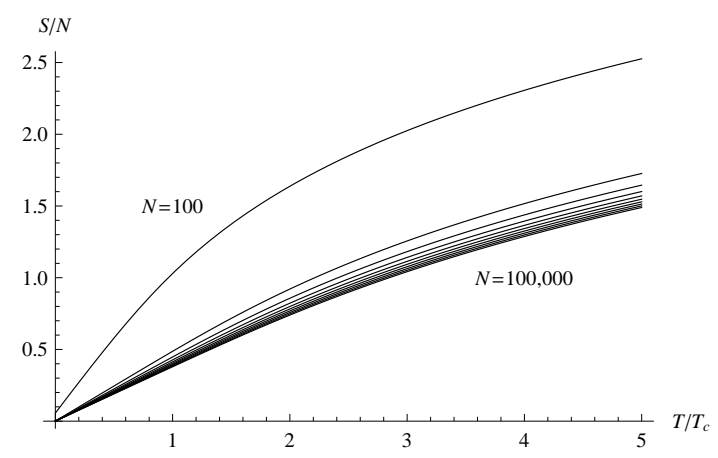

FIG. $4: S / \mathcal{N}$ as a function of $T / T_{c}$.

Expanding $\ln \Xi$ in a power series and applying the transformation in (42) we arrive at

$$
\ln \Xi=-\beta M_{0}-\ln (1-\lambda)+\frac{\operatorname{Li}_{2}(\lambda)}{\beta \hbar / l}
$$

and combine this expression with (54). Then the entropy function can be given in terms of the ratio $x=T / T_{c}$ as

$$
S(\beta, \mathcal{N})=\frac{2 x \mathcal{N}}{\mathcal{W}_{N}} \operatorname{Li}_{2}(\lambda)-\ln (1-\lambda)-\mathcal{N} \ln \lambda
$$

The specific entropy is shown in figure 4 for $\mathcal{N}$ ranging from one hundred to one hundred thousand shells. It is seen to decrease with increasing $\mathcal{N}$. In the limit of low temperatures, i.e., as $x \rightarrow 0$, the leading behavior of the entropy is as

$$
S \approx \frac{\pi^{2}}{2 \beta \hbar / l}+\ln \mathcal{N}+1
$$

This is not vanishing, but the specific entropy, $S / \mathcal{N}$, does vanish in the thermodynamic limit as the temperature approaches zero and so it is consistent with a generalized version of the third law. If we now consider this function in terms of its natural variables, $M$ and $\mathcal{N}$ we find from (54) that in this same limit

$$
\frac{\pi}{\beta \hbar / l} \approx \sqrt{\frac{6\left(M-M_{0}-\mathcal{N} \varepsilon_{0}\right)}{\hbar / l}},
$$

showing that the black hole temperature vanishes as the black hole mass approaches $M_{0}+$ $\mathcal{N} \varepsilon_{0}$, at which point the entropy approaches $\ln \mathcal{N}$. In fact, written in terms of the black hole mass and the number of shells, the entropy is

$$
S \approx \frac{\pi}{3} \sqrt{\frac{6\left(M-M_{0}-\mathcal{N} \varepsilon_{0}\right)}{\hbar / l}}+\ln \mathcal{N}+1 .
$$

Small $x$ is the regime in which Bose condensation plays a dominant role. At temperatures well below the critical temperature, where a large fraction of the shells are in the ground state, the first term on the right becomes negligible and the entropy is dominated by its 


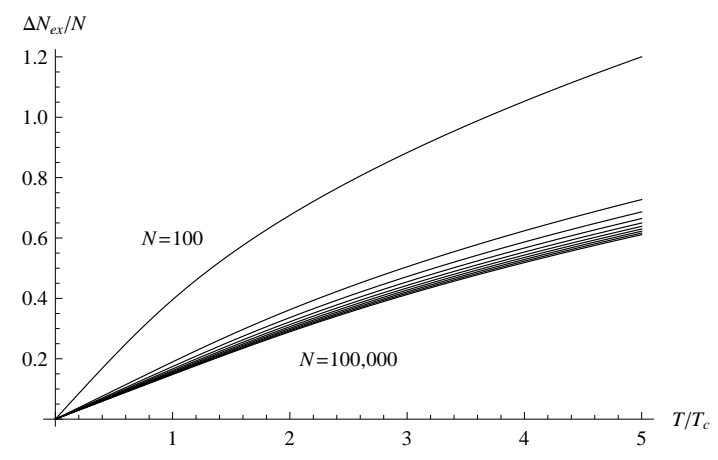

FIG. 5: $\Delta N_{\mathrm{ex}} / \mathcal{N}$ as a function of $T / T_{c}$.

logarithmic behavior. Thus even though a quantization of Einstein's gravity yields the usual black hole results in the thermodynamic limit and at high temperatures, its behavior outside this regime may present interesting features peculiar to quantum statistics. In particular, we see that BTZ black holes turn cold once condensation sets in. This is the "small" black hole or short distance regime in $2+1$ dimensions.

Finally, for completeness, we address the mean square fluctuations. We are interested in evaluating

$$
\Delta N_{\mathrm{ex}}^{2}=-\frac{1}{\beta} \sum_{j=1}^{\infty} \frac{\partial N_{j}}{\partial \varepsilon_{j}}=\sum_{j=1}^{\infty} \sum_{r=1}^{\infty} r \lambda^{r} e^{-\beta \hbar r j / l} .
$$

The sums can be evaluated with the help of the representation in (42) and one obtains

$$
\Delta N_{\mathrm{ex}}^{2}=\frac{\mathrm{Li}_{2}(\lambda)}{(\beta \hbar / l)^{2}} .
$$

The fluctuations are plotted as a function of $x=T / T_{c}$ in figure 5 for one hundred, one thousand and one hundred thousand shells. The uppermost curve represents $\mathcal{N}$ ranging from one hundred to one hundred thousand shells. and the fluctuations are seen to decrease with $\mathcal{N}$. Near the critical temperature, they fall off as $1 / \ln \mathcal{N}$.

\section{DISCUSSION}

Although well adapted to problems possessing high symmetry such as in cosmology and spherical collapse, the canonical approach suffers from several ambiguities. We have dealt with them explicitly before arriving at the results quoted in the introduction, but only within the context of the specific class of models with which we work. Thus canonical quantization cannot be expected to yield the final theory of quantum gravity as it stands, but its results should be taken seriously for models in which the ambiguities can be resolved. One advantage of our approach to the quantum properties of black holes is that the fundamental degrees of freedom and the black hole microstates are given a transparent meaning. Another is that the same picture holds for higher dimensional black holes in Einstein's general relativity both with and without a cosmological constant.

It seems remarkable at first sight that the spherical midisuperspace models of collapse should yield the Bekenstein-Hawking entropy of the black hole, considering that all non- 
spherically symmetric states have been eliminated by the symmetry reduction and therefore do not contribute to the entropy. We cannot definitively tell why this is so, but we speculate that non-spherically symmetric states may not describe a regular horizon (with constant surface gravity) and so do not contribute to the entropy of a static black hole. However, this conjecture requires further clarification and the question seems worthy of further investigation.

The point we have made in this paper is that the statistics applied in counting black hole microstates, together with the specific models of the microstates, can play a crucial role in the behavior of a black hole at low temperatures and outside the thermodynamic limit. We have demonstrated this explicitly in the case of the BTZ black hole for which Bose statistics must be applied to correctly recover its thermodynamic properties. We have argued that the BTZ black hole condenses in the sense that there is a critical temperature below which a significant fraction of the black hole mass is in the ground state.

Our results can be summarized as follows: (i) the condensation temperature grows almost linearly with the number of shells and can be quite large, (ii) the specific heat grows linearly below the condensation temperature but slowly above it, approaching unity in the limit of very high temperatures and (iii) the Bekenstein-Hawking area law is obeyed only in the thermodynamic limit, outside of which the entropy deviates from the area law, approaching $\ln \mathcal{N}$ in the limit as $T \rightarrow 0$, which occurs at finite mass. According to the results of [36], this would signal a breakdown of general relativity at low enough temperatures. This seems to be an interesting direction for future research.

Of considerable phenomenological importance of course is the question of whether these results are reproduced by realistic black holes in higher dimensions and precisely how. If they are, then Bose condensed primordial black holes would make natural Dark Matter candidates. We will examine the consequences of the Bose condensation described in this paper for gravity at short distances in a future publication.

\section{Acknowledgements}

LCR Wijewardhana was supported in part by the U.S. Department of Energy Grant No. DE-FG02-84ER40153.

[1] J. D. Bekenstein, Ph.D. thesis, Princeton University (1972);

ibid, Lett. Nuovo Cimento 4 (1972) 737;

ibid, Phys. Rev. D 7 (1973) 2333.

[2] J. M. Bardeen, B. Carter, and S. W. Hawking, Comm. Math. Phys. 31 (1973) 161.

[3] S. W. Hawking, Comm. Math. Phys. 43 (1975) 199.

[4] A. Strominger and C. Vafa, Phys. Lett. B 379 (1996) 99.

[5] G.T. Horowitz and J. Polchinski, Phys. Rev. D 55 (1997) 6189.

[6] A. Dabholkar, Phys. Rev. Lett. 94 (2005) 241301.

[7] S. Hyun, W.T. Kim, and J. Lee, Phys. Rev. D 59 (1999) 084020.

[8] R. Emparan, JHEP 9906 (1999) 036. 
[9] S. W. Hawking, J. Maldacena, and A. Strominger, JHEP 0105 (2001) 001.

[10] S. A. Mukherji and S. S. Pal, JHEP 0205 (2002) 026.

[11] S. K. Chakrabarti, K.S. Gupta, and S. Sen, Int. J. Mod. Phys. A23 (2008) 2547.

[12] C. Rovelli, Phys. Rev. Lett. 77 (1996) 3288.

[13] A. Ashtekar, J. Baez, A. Corichi, and K. Krasnov, Phys. Rev. Lett. 80 (1998) 904.

[14] M. Domagala and J. Lewandowski, Class. Quant. Grav. 21 (2004) 5233.

[15] K. A. Meissner, Class. Quant. Grav. 21 (2004) 5245.

[16] A. Corichi, J. Diáz-Polo and E. Fernández-Borja, Phys. Rev. Lett. 98 (2007) 181301.

[17] I. Agulló, J. F. Barbero G., J. Diáz Polo, E. Fernández-Borja and E. J. S. Villaseňor, Phys. Rev. Lett. 100 (2008) 211301.

[18] C. Kiefer and G. Kolland, Gen. Rel. Grav. 40 (2008) 1327.

[19] C. Vaz and L. Witten, Phys.Rev. D63 (2001) 104020.

[20] M. Bañados, C. Teitelboim and J. Zanelli, Phys. Rev. Lett. 72 (1994) 957.

[21] C. Vaz, S. Gutti, C. Kiefer and T.P. Singh, Phys. Rev. D 76 (2007) 124021.

[22] C. Vaz, S. Gutti, C. Kiefer, T.P. Singh and L.C.R. Wijewardhana, Phys. Rev. D 77 (2008) 064021.

[23] C. Vaz and L.C.R. Wijewardhana, Phys. Rev. D 79 (2009) 084014.

[24] S. Hyun, J. Kor. Phys. Soc. 33 (1998) S532.

[25] K. Sfetsos and K. Skenderis, Nucl. Phys. B517 (1998) 179.

[26] R. B. Mann and S. F. Ross, Phys. Rev. D 47, 3319 (1993).

[27] S. Gutti, Class. Quant. Grav. 22 (2005) 3223.

[28] C. Vaz, L. Witten, T.P. Singh, Phys.Rev. D 69 (2004) 104029.

[29] C. Kiefer, K. Mueller-Hill, C. Vaz, Phys.Rev. D 73 (2006) 044025.

[30] G. Hardy and S. Ramanujan, Proc. Lond. Math. Soc. 17 (1918) 75.

[31] M. Bañados, M. Henneaux, C. Teitelboim, and J. Zanelli, Phys. Rev. D 48 (1993) 1506.

[32] Martin Holthaus, Eva Kalinowski and Klaus Kirsten, "Condensate Fluctuations in Trapped Bose Gases: Canonical vs. Microcanonical Ensemble", arXiv:cond-mat/9804171]

[33] F. Dalfovo, S. Giorgini, L.P. Pitaevskii and S. Stringari1, Rev. Mod. Phys. 71 (1999) 463.

[34] W. Ketterle and N.J. van Druten, Phys. Rev. A 54 (1996) 656.

[35] G-L. Ingold and A. Lambrecht, Eur. Phys. Jour. D 1 (1998) 29.

[36] T. Jacobson, Phys. Rev. Lett. 75 (1995) 1260. 